\title{
Bayesian Probability-based UI Control Framework of Sexual Violence Prevention Education Apps
}

\author{
Yunsick Sung ${ }^{1}$, Donguk Kim ${ }^{1}$, Hyung Jin Park ${ }^{2}$, Kyung Min Park ${ }^{3, *}$ \\ ${ }^{1}$ Faculty of Computer Engineering, Keimyung University, Daegu 704-701, South Korea, \\ ${ }^{2}$ College of Fine Art, Keimyung University, Daegu 704-701, South Korea, \\ ${ }^{3}$ College of Nursing, Keimyung University, Daegu 704-701, South Korea, \\ \{yunsick, donguk, phj, kmp\}@kmu.ac.kr
}

\begin{abstract}
This paper proposes a UI control method for increasing the learning effect of sexual violence prevention education Apps. By considering the frequency and properties of UI elements, UI elements are modified dynamically. Therefore, the learning effect of the apps could be improved.
\end{abstract}

Keywords: UI framework, Sexual violence, Bayesian probability.

\section{Introduction}

With recent changes in sex-related phenomena and values in the society as a whole, sex-related problems have emerged as a serious issue [1]. Especially of late, as distorted sexual culture is encountered in personal spaces, such as cyberspace, sexual crimes such as sexual harassments, sexual abuse, sexual assault, stalking, and cyber sexual violence are growing in number [2]. In particular, for changes in sexual attitude during primary school years, it is necessary for primary school students to know the actual situation that is the unique circumstance of sexual violence and provide permissive conditions that allow open questions and discussions on subjects of curiosity. Moreover, an alternative is needed to receive more creative education beyond the existing format. However, sex education media developed in South Korea consist mostly of computer-based media, such as CDs or animation. Especially, to prevent sexual violence in primary school students, development of media that enable primary school students to engage in interactive learning is desperately needed.

This paper proposes a UI control framework of education apps for protecting sexual violence of elementary school students. The learning effect of the education apps can be improved by reformatting static UI elements through UI control framework.

This paper is consisted as follow. Section 2 proposes a UI control framework. Section 3 shows the result of the proposed method. Finally Section 4 concludes.

\footnotetext{
* Corresponding Author: Kyung Min Park (kmp@kmu.ac.kr)
} 
Advanced Science and Technology Letters

Vol.106 (Information Technology and Computer Science 2015)

\section{UI Framework}

The proposed UI framework increases the learning effect of education apps by changing the properties of UI elements such as size or color. The UI elements that will be changed should be chosen first as follow and then modified.

First, the frequency of utilized UI elements is considered. Given that the proposed framework induces the desired frequency of UI elements, the frequency is the most important factor. To measure the frequency of the ith UI elements, Bayesian probability is applied as shown in $\mathrm{Eq}(1)$ where $n$ is the number of UI elements. $p_{i}$ is the Bayeisan probability[3] of the ith UI element. $\mathrm{c}_{\mathrm{i}}$ is the number of the utlization of the ith UI elements.

$$
p_{i}=\frac{c_{i}}{\sum_{j=1}^{n} c_{j}}
$$

Next, the importance of utilized UI elements is considered. $\mathrm{w}_{\mathrm{i}}$ is the weight of the ith UI element. Weights are determined depending on how important each element is. As a result, each importance $v_{i}$ of elements is calculated with $\mathrm{Eq}(2)$ where $\mathrm{m}$ is the number of weights.

$$
v_{i}=p_{i} \times w_{1} \times w_{2} \times \ldots \times w_{m} .
$$

After determining the importance of elements, the UI elements that will be changed is selected considering the importance. The number of selected UI elements is determined depending on contents.

\section{Experiment}

In the experiment, the proposed framework was applied to the app "Sexual violence, how should I do?" The app is consisted of three stages: Title stage, map stage, and scenario stage.
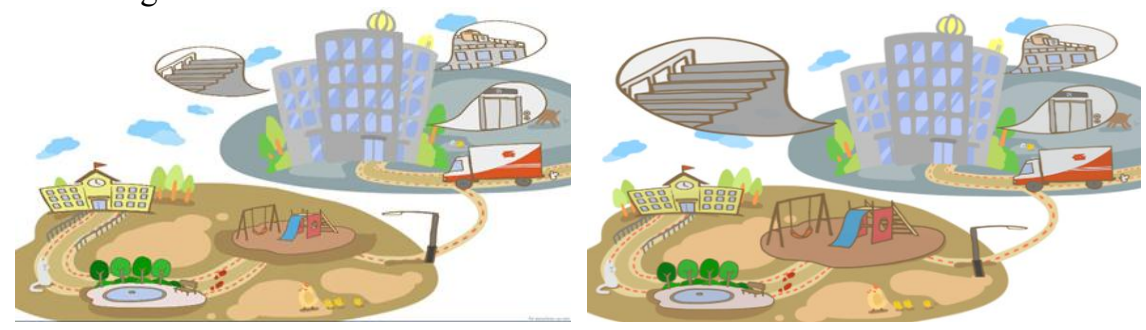

Fig. 1. In map stage, there are eight buttons for school, park, playground, alley, delivery, elevator, rooftop, stairs scenarios. Left shows the default UI in map stage. Right shows the changed UI in map stage by applying UI control framework.

Map stage provides the map where users can select one of scenarios given that the buttons in the map stage are linked with the corresponding scenarios. When any button is clicked, the corresponding scenario of the clicked button is played in 
scenario stage. UI control framework handles the properties of the buttons in map stages. By changing the size of the button, users are induced to click the resized buttons.

To recognize how subjects click buttons in map stage and which buttons subjects prefer, each subject has three chances to click it. There are eight subjects. Table 1 shows the result of clicked buttons and Table 2 shows the rank of clicked buttons.

Table 1. Subjects' clicked buttons in the default UI of map stage. Subjects clicked buttons three times in order.

\begin{tabular}{|c|c|c|c|}
\hline & 1st Selection & 2nd Selection & 3rd Selection \\
\hline Subject 1 & Playground & Elevator & Rooftop \\
\hline Subject 2 & Delivery & Playground & Stairs \\
\hline Subject 3 & School & Park & Elevator \\
\hline Subject 4 & Playground & Park & Delivery \\
\hline Subject 5 & Playground & School & Park \\
\hline Subject 6 & School & Park & Playground \\
\hline Subject 7 & School & Park & Playground \\
\hline Subject 8 & Playground & Park & Delivery \\
\hline
\end{tabular}

Table 2. The rank of clicked buttons. Playground was the 1st button based on the number of button clicks. School and park were the 2nd and 3rd buttons.

\begin{tabular}{|c|c|c|c|c|c|c|c|c|}
\hline & $\begin{array}{c}\text { Play } \\
\text {-ground }\end{array}$ & School & Park & Delivery & Elevator & Rooftop & Stairs & Alley \\
\hline Clicks & 7 & 4 & 6 & 3 & 2 & 1 & 1 & 0 \\
\hline
\end{tabular}

The buttons were chosen by applying two weights as shown in Table $3 . \mathrm{w}_{1}$ and $\mathrm{w}_{2}$ are set by considering where sexual violence are invoked by whom people know and where children sexual violence are happen frequently.

Table 3. Weights and Ranks. When the proposed framework was applied, two weights were utilized. As a result, playground and stairs were selected.

\begin{tabular}{|c|c|c|c|c|c|c|c|c|}
\hline & Playground & School & Park & Delivery & Elevator & Rooftop & Stairs & Alley \\
\hline $\mathrm{p}_{\mathrm{i}}$ & 0.29 & 0.16 & 0.25 & 0.125 & 0.08 & 0.04 & 0.04 & 0 \\
\hline $\mathrm{w}_{1}$ & & 2 & & & & 2 & 2 & 2 \\
\hline $\mathrm{w}_{2}$ & 4 & & & 2 & & & 4 & \\
\hline $\mathrm{v}_{\mathrm{i}}$ & 1.16 & 0.32 & 0.25 & 0.25 & 0.08 & 0.08 & 0.32 & 0 \\
\hline Rank & 1 & 2 & 4 & 4 & 5 & 5 & 2 & 6 \\
\hline
\end{tabular}


Advanced Science and Technology Letters

Vol.106 (Information Technology and Computer Science 2015)

By applying the proposed framework, the buttons in map stage was changed as shown in Fig. 1(Right). The button sizes of playground and stairs were increased. Table 4 shows the 2 nd result based on the changed buttons. Before changing the size of the buttons, two buttons were chosen about 33\%. However, after applying the proposed framework, those were chosen up to $50 \%$ even though only two buttons were resized.

Table 4. The rank of clicked buttons. Playground was the 1st button based on the number of clicked buttons. School and park were the 2 nd and 3rd button.

\begin{tabular}{|c|c|c|c|}
\hline & 1 & 2 & 3 \\
\hline Subject 1 & Playground & Elevator & Rooftop \\
\hline Subject 2 & Rooftop & Stairs & Elevator \\
\hline Subject 3 & Stairs & Playground & Rooftop \\
\hline Subject 4 & Playground & Stairs & Park \\
\hline
\end{tabular}

\section{Conclusion}

This paper proposed a UI control framework for increasing the learning effects of sexual violence prevention education apps. Given that users usually do not play provided whole scenarios, it is required to let users play some of the scenarios that are more effective than other scenarios. By changing the size of UI buttons, the possibility when users click the button of more effective scenarios was increased.

Acknowledgement. This research was supported by Basic Science Research Program through the National Research Foundation of Korea(NRF) funded by the Ministry of Education(NRF-2015009659)

\section{References}

1. Lee, K., Do, M.: The Effectiveness of the Child Sexual Violence Prevention Coaching Programs for Children into the Children /'s Sexual Knowledge and the ability of Coping with Sexual Matters and Violences. Korean Journal of Parent Education Research, 8(1), 45-67 (2011)

2. Ministry of Gender Equality \& Family.: Youth Harmful Environmental Survey, (2012)

3. Berger, J. O.: Statistical Decision Theory and Bayesian Analysis. Springer Series in Statistics, Springer-Verlag (1985) 